\title{
Population distribution and urbanization on both sides of the Hu Huanyong Line:
} Answering the Premier's question

\author{
CHEN Mingxing ${ }^{1}$, GONG Yinghua ${ }^{1,4}$, LI Yang ${ }^{3}$, LU Dadao ${ }^{1}$, 'ZHANG Hua ${ }^{2}$ \\ 1. Institute of Geographic Sciences and Natural Resources Research, Key Laboratory of Sustainable Regional \\ Development and Modeling, CAS, Beijing 100101, China; \\ 2. State Key Laboratory of Remote Sensing Science, School of Geography, Beijing Normal University, Beijing \\ 100875, China; \\ 3. China Center for Modernization Research, CAS, Beijing 100190, China; \\ 4. University of Chinese Academy of Sciences, Beijing 100049, China
}

\begin{abstract}
In late November 2014, while attending a science exhibition on human settlement at the National Museum, Premier Li Keqiang posed a question to society and scientists regarding the Hu Huanyong Line, which the media subsequently dubbed "the Premier's question". This increased awareness and interest in the Hu Huanyong Line and launched a lively debate which provoked a variety of views. In an attempt to address the Premier's question, this paper firstly reviews the origins of the Hu Huanyong Line, named after the famous population geographer who proposed it in 1935 as part of a wider debate on domestic overpopulation. Using demographic data from China's first, fifth and sixth censuses, as well as the ArcGIS platform, we analyze the size, proportion and density of populations in the areas southeast and northwest of the Hu Huanyong Line, showing that urbanization and migration have not changed the pattern of population distribution observed by Hu Huanyong. Based on this, we suggest that the pattern of a dense population southeast of the line and sparse population northwest of the line will not fundamentally change for a relatively long time, nor will the situation of urban agglomerations being mainly found in the southeastern region. We also argue that climate and other physical geographic conditions determine that the Hu Huanyong Line shall remain in place. We believe that the question posed by Premier Li Keqiang is solvable, and that with positive policy guidance and rational spatial organization, the northwestern region can achieve more modernization and better quality urbanization, while the same is true for the central region.
\end{abstract}

Keywords: Hu Huanyong Line; Premier's question; population distribution; urbanization; patterns; trends

Received: 2016-01-20 Accepted: 2016-04-15

Foundation: National Natural Science Foundation of China, No.41671125, No.41601121; Programme of Bingwei Excellent Young Scientists of the Institute of Geographic Sciences and Natural Resources Research, CAS, No.2015RC202; National Science and Technology Support Program, No.2012BAJ15B02

Author: Chen Mingxing (1982-), PhD and Associate Professor, specialized in urbanization and regional development. E-mail: chenmx@igsnrr.ac.cn

"Corresponding author: Zhang Hua (1979-), Lecturer, E-mail: zhanghua@bnu.edu.cn 


\section{Introduction}

At a science exhibition on human settlement at the National Museum in late November 2014, Premier Li Keqiang pointed to the Hu Huanyong Line on a map of China and stated that $94 \%$ of China's population lives in $43 \%$ of its land area, and that the line visually demonstrates the reality of China having a densely populated southeast and sparsely populated northwest. But, he said, the central and western regions need to urbanize just as the eastern coastal region has done. He went on to say that China is a multi-ethnic country with a vast territory, so it is necessary to consider how to break with this traditional demographic pattern through overall planning and coordinated development in order for people in central and western regions to benefit from modernity (Yang, 2014). On the back of this, journalists have referred to the question of how to break the pattern of the Hu Huanyong Line as "the Premier's question". The Premier's speech at the exhibition has since become a hot topic in the media, society and among local governments, while some academics have held lively discussions and put forward a host of views on the matter. A commonly held view is that the traditional demographic pattern of the Hu Huanyong Line can be broken. Although the line has to date been a dividing line of urbanization to some extent, it is felt that China's new-type urbanization provides an opportunity to break the mold of the Hu Huanyong Line, and with the trends of suburbanization and counter-urbanization, it is possible to make the most of the situation to break with the traditional pattern of the Hu Huanyong Line, as suggested in the Premier's question (Li, 2015). What is more, implementation of the Belt and Road Initiative will help break the pattern of the Hu Huanyong Line, boosting the resource rent and locational advantages of the western region, directly opening up the vast area west of the Hu Huanyong Line, which will rapidly create room for the economy to develop and encourage flows of people and goods into the region (Kuang, 2014). A different view is that the traditional demographic pattern represented by the Hu Huanyong Line cannot be altered. Advocates of this viewpoint argue that $95 \%$ of China's population lives southeast of the line due to physical geographic conditions and that there is no chance this population distribution will change in the foreseeable future (Jia, 2014). Yet another view is what could be referred to as a conditional breaking of the traditional pattern. A simulation study of the Hu Huanyong Line has suggested that under certain conditions owing to climate change China's western region could receive 40 million people, which depends on two factors: first, the development of new industries led by the information economy and, second, the development of a hub-network form of spatial organization, wherein the country is treated as a single entity and there are no isolated areas (Wang, 2014).

$\mathrm{The} \mathrm{Hu}$ Huanyong Line is the result of a classic theory in Chinese geography and named after the famous population geographer, Hu Huanyong, who proposed it for the first time in a study entitled Distribution of China's population $(\mathrm{Hu}, 1935)$ to demonstrate the different population densities of the areas either side of the line, which he originally called the Aihui-Tengchong Line (the line ran from the city of Aihui in the northeast to Tengchong county in the southwest), later becoming the Heihe-Tengchong Line after the former city was renamed. On the 100th anniversary of the founding of modern geography in China, the Geographical Society of China and the Chinese National Geography magazine jointly launched the "Century of Great Chinese Geographical Discoveries" event, and selected the 
Hu Huanyong Line as one of China's 30 great geographical discoveries. Others on the list were Hou Renzhi for his planning and development of Beijing, Zhou Lisan and Wu Chuanjun for their agricultural zoning program, and Lu Dadao for his T-shaped spatial strategy for regional development based on his theory of the "pole-axis" system.

The first time we heard of "the Premier's question" was a year before the current debate broke out. At an urbanization research review meeting held by the Chinese Academy of Sciences (CAS) and the Chinese Academy of Engineering (CAE) in Meeting Room 1 at Zhongnanhai in August 2013, Lu Dadao, representing the urbanization project team from CAS, delivered a half-hour speech entitled "Explanation and Suggestion of an Urbanization Path Suited to China's National Conditions", in which he emphasized the concept of an urbanization path that fits China's national conditions and that has Chinese characteristics, and clearly stated that China's future urbanization should not be excessive. The authors of this paper were also present at that meeting, during which Premier Li Keqiang said in his speech, "Urbanization directly relates to the success or failure of China's modernization. Should we, and can we, break with the pattern of the Hu Huanyong Line? Please help to research this question."

The older generation of geographers taught us that geography is the science of statecraft (Lu, 2011), human and economic geography face the main battlefield of the national economy, and researching the hot topics in economic and social development is an important characteristic of China and the source of the vitality of human and economic geography in China. As such, to address the current difficulties in understanding the Hu Huanyong Line, this paper shall first review its origins and use data from the first, fifth and sixth censuses to analyze population distribution on either side of the line, on the basis of which we will then focus on analyzing "unbreakable" aspects of the Hu Huanyong Line and the "solvable" features of the Premier's question. The Hu Huanyong Line is an objective geographical law that cannot be "broken", but there is a solution to the core issue raised by the Premier. On the basis of the following objective laws, humans are capable of rationally distributing people, towns and cities, and industries to achieve (even) higher-level urbanization and modernization in China's central and western regions, and thus attempt to answer and explain the Premier's question and provide a possible answer for society to explore. This paper hopes to get the ball rolling in this area and welcomes contributions by others.

\section{Methodology and data sources}

\subsection{Research method}

This paper is a comprehensive analysis of the recently popular topic referred to in the media as "the Premier's question". Simply speaking, the key point of this article is that the Hu Huanyong Line cannot be "broken". It is an objective geographical law that must be followed and cannot be overcome or transgressed by humans. At the same time, however, the actual query contained in the Premier's question does have a solution. The issue that the Premier is actually interested in is the urbanization of the central and western regions of China, and this paper analyzes the western region's urbanization path to show that a solution exists to the Premier's question. With this objective in mind, this paper shall, first of all, un- 
dertake a quantitative analysis of the scale, density and proportion of the populations either side of the Hu Huanyong Line for the period 1933 to 2010, looking at the county scale and using the ArcGIS analysis methods with four time sections. On the basis of this, the focus shall shift to a comprehensive analysis of the regularity of the Hu Huanyong Line and the urbanization path of the western region.

From an analysis of the basic pattern of the Hu Huanyong Line, namely a densely populated east and sparsely populated west, and its evolution, as well as the relationship between the $\mathrm{Hu}$ Huanyong Line and the distribution pattern of urban agglomerations, we illustrate the features that make the line "unbreakable" and the reasons why it is in terms of its physical geographic elements. We select the four indicators of the eco-environment, terrain, climate, and land and resources to analyze population distribution either side of the line. The eco-environment element is based on results of national ecological function zoning; terrain is based on an analysis of the relationship between digital elevation models (DEM) and population distribution; climate is based on an analysis of annual average rainfall; land and resources reflect the distribution of national arable land. After determining the evaluation factors and their corresponding indicators, a conceptual model of the unbreakable Hu Huanyong Line population distribution was constructed:

$$
P=f(T, C, E, L \ldots)
$$

where $P$ is population distribution, $T$ is terrain, $C$ is climate, $E$ is environment and $L$ is land and resources. In fact, population distribution is a complex geographic phenomenon affected by various elements of physical geography as well as elements of human and economic geography. Due to the limitations of the data collected and the different foci of this study, this paper selects the four most representative physical geographic elements from which to construct an analytical conceptual model, and discusses the relationship between natural elements and the spatial distribution of population in order to explain the underlying reasons the traditional demographic pattern of the Hu Huanyong Line cannot be broken.

\subsection{Data sources}

This study uses population data taken from previous censuses and sampling survey data. Data on urbanization and urban agglomerations are taken from the China Statistical Yearbook, China City Statistical Yearbook and other almanacs. Information on terrain comes from the National Geomatics Center's 90 m DEM. Climate data is based on average annual rainfall figures collected by the China Meteorological Administration. Environmental data is taken from a paper published by the Ministry of Environmental Protection and the Chinese Academy of Sciences titled "National Ecological Function Zoning". Land and resources data is taken from a changing land use survey by the Ministry of Land and Resources.

\section{The Hu Huanyong Line and population distribution changes}

\subsection{Origin and significance of the Hu Huanyong Line}

An important geographic feature of China's population distribution is its regional imbalance. Despite having been this way throughout China's history, it was not until 1935 and the publication of Hu Huanyong's study entitled Distribution of China's population in Acta Geo- 
Geographica Sinica that the specific quantitative facts were presented. Based on diagrams showing China's population distribution and density, Hu proposed the now-famous demographic boundary, running from Aihui (renamed Heihe in 1983) in Heilongjiang Province to Tengchong in Yunnan Province, known nowadays simply as the Hu Huanyong Line.

Hu's initial study emerged amid a similar backdrop to that of the current debate. The paper's introduction considered whether China was overpopulated and whether wide-scale immigration might take place. He pointed out the need for a precise understanding of China's population distribution in order to arrive at answers to these questions ( $\mathrm{Hu}, 1935)$. The paper, therefore, was published at a time of great debate regarding China's population. On the question of whether China is overpopulated, two schools of thought existed. The first argued that China is overpopulated, making it difficult to earn a living and resulting in severe unemployment and mass immigration abroad. The second argued that China is not overpopulated and that its average population density is dissimilar to that of Western Europe. In order to address this hotly debated issue, Hu collected the limited population data available at the time for every region of China down to the county level and drew up diagrams showing China's population distribution and density, which showed a stark pattern.

In his study, Hu divided China's population density into eight levels. The first level had more than 400 people per $\mathrm{km}^{2}$ and the eighth level had less than 1 person per $\mathrm{km}^{2}$. In his explanation of the eighth level of population density, $\mathrm{Hu}$ stated, "If one draws a line from Aihui in Heilongjiang southwest to Tengchong in Yunnan, dividing the country into southeast and northwest parts, the area of the southeast part is 4 million $\mathrm{km}^{2}$, accounting for approximately $36 \%$ of the country's total, while that of the northwest part is 7 million $\mathrm{km}^{2}$, accounting for $64 \%$ of the country's total; however, in terms of population distribution, the southeast part contains 440 million people, being $96 \%$ of the country's total, while the northwest part contains only 18 million, $4 \%$ of the country's total, thus illustrating the great disparity between them."

The Hu Huanyong Line is an approximate geographic boundary of population, but due to technical restrictions at the time, it does not have a fixed geographic location, which is perhaps the reason the map included in the study did not have the line drawn on it. It is generally believed, however, that the $\mathrm{Hu}$ Huanyong Line lies at an approximate angle of $45^{\circ}$ between Aihui in Heilongjiang and Tengchong in Yunnan, dividing China into northwest and southeast sections. Based on this location of the line, the southeast section, made up of plains, river and lake networks, rolling hills, karst topography and Danxia landforms, and with an economic foundation of agriculture stretching back to ancient times, accounts for $36 \%$ of China's total land area and $96 \%$ of its population. The northwest section, however, made up of grasslands, deserts and snow-covered plateaus, and dominated by nomadic cultures, accounts for $64 \%$ of China's land area and only $4 \%$ of its population. Hu looked at diagrams of topography, rainfall and the distribution of ethnic groups in his paper and noticed an extremely close relationship between them and the population distribution. In the final section of his study, Hu gave a detailed description of sparsely populated areas of the southeast side of the line and the densely populated areas of the northwest side of the line, from which he further analyzed the population distribution in China at the time and the geographic features of the extreme population imbalance. 
Hu's classic study is of special significance. It clearly described the spatial distribution of China's population for the first time, as well as pointing out the existence of geographical differences in natural conditions and economic activity and fully explaining the research features of geographic comprehensiveness on integration, regional differences and spatial distribution. The Hu Huanyong Line clearly delineated the disparity in population density between the southeast and the northwest, and it is one of the most concise ways of portraying the spatial distribution of China's population, which has been acknowledged by numerous domestic and international scholars (Wu, 2001; Liu and Wang, 2001; Ding et al., 2005; Feng et al., 2014; Ge and Feng, 2008; Wu and Wang, 2008; Wang, 1997; Wang, 1998; Shen, 1982; Liu, 2014; Zhu, 2006; Jia and Su, 2015; Liu, 2004 ). Following further research in various fields, it has been given implications far beyond the sphere of influence of population geography, or even human geography. Interestingly, the $\mathrm{Hu}$ Huanyong Line was not originally the focus of Hu's classic study, but this important boundary in population geography aroused the interest of so many scholars that it later became an important point of reference for scholars and decision makers. Having been widely recognized and referenced by Chinese and international demographers and geographers ever since, many years later, the line was referred to as the "Hu Huanyong Line" by Professor Tian Xinyuan of Ohio State University (Tien 1981, 1973).

\subsection{Changes in population distribution on both sides of the Hu Huanyong Line}

This year is the 80th anniversary of the idea of the Hu Huanyong Line. In those 80 years, China's society and economy have undergone enormous changes, particularly rapid urbanization and large-scale migration, which begs the question whether the line still perfectly represents the regional differences in China's population distribution. In this section, data from the first, fifth and sixth censuses, as well as ArcGIS software, are used to analyze the density of populations in China's county-level administrative districts, in order to verify whether urbanization and migration have changed the pattern of population distribution observed by $\mathrm{Hu}$ Huanyong.

Using the Hu Huanyong Line drawn between the city of Heihe in the north and Tengchong county in the south, China's territory is divided into a southeast portion and a northwest portion. Any counties bisected by the line are determined to be either in the southeast or northwest based on which side of the line the county government is located in. Data from the first, fifth and sixth censuses have been used to produce diagrams showing China's population density in 1953, 2000 and 2010 (Figure 1) and compare the population distributions of the areas southeast and northwest of the line (Table 1).

Table 1 The change of population proportion on two sides of the Hu Huanyong Line (1933-2010)

\begin{tabular}{|c|c|c|c|c|c|c|}
\hline \multirow[b]{2}{*}{ Year } & \multicolumn{3}{|c|}{ Southeast } & \multicolumn{3}{|c|}{ Northwest } \\
\hline & Area $(\%)$ & $\begin{array}{l}\text { Population } \\
(\%)\end{array}$ & $\begin{array}{c}\text { Population } \\
\text { density } /\left(\mathrm{km}^{2}\right)\end{array}$ & Area $(\%)$ & $\begin{array}{l}\text { Population } \\
(\%)\end{array}$ & $\begin{array}{c}\text { Population } \\
\text { density } /\left(\mathrm{km}^{2}\right)\end{array}$ \\
\hline 1933 & 36.00 & 96.00 & 135.39 & 64.00 & 4.00 & 5.03 \\
\hline 1953 & 43.24 & 94.80 & 139.51 & 56.76 & 5.20 & 5.83 \\
\hline 2000 & 43.24 & 94.59 & 303.78 & 56.76 & 5.41 & 13.23 \\
\hline 2010 & 43.24 & 94.41 & 325.84 & 56.76 & 5.59 & 14.68 \\
\hline
\end{tabular}




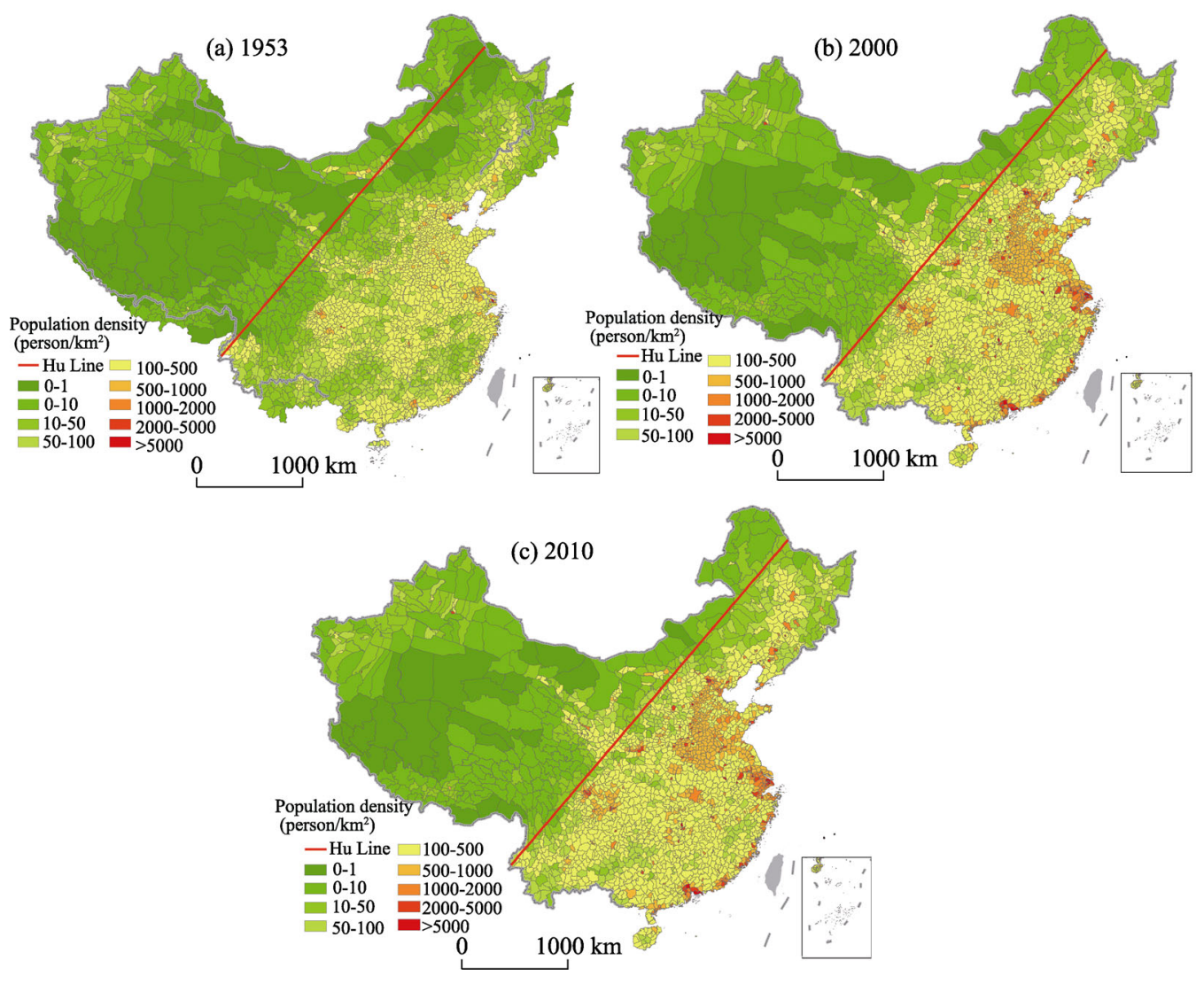

Figure 1 The change of population proportion on two sides of the Hu Huanyong Line since 1953

Between 1953 and 2010, population density increased significantly in the majority of China, but the spatial pattern of population density remained largely static. In that time, China's total population increased rapidly from 602 million in 1953, to 1.29 billion in 2000, to 1.34 billion in 2010. National average population density also increased considerably, from $62.7 / \mathrm{km}^{2}$ in 1953 , to $134.93 / \mathrm{km}^{2}$ in 2000 , to $139.55 / \mathrm{km}^{2}$ in 2010 , which is reflected in the significant increases in population density in the majority of areas in the population density diagram. However, areas with high population density have always been located in the middle and lower reaches of the Yellow River, the Yangtze River Delta, the Sichuan Basin and the southeast coastal areas, with clear differences in population density evident between the southeast and northwest parts. The Hu Huanyong Line thus clearly delineates the pattern of China's population density.

In order to further compare population distribution either side of the Hu Huanyong Line, we calculated the percentage of the total population living either side of the line (Table 1). It should be noted that, due to the imprecise positioning of the $\mathrm{Hu}$ Huanyong Line, there are slight differences in the calculations of different scholars. But this does not affect our analysis, and the same criteria were used in this study for the calculations of each of the three years, making comparability easier and giving greater reliability. According to the calculations of the Hu Huanyong himself, in 1933 36\% of China's land area and $96 \%$ of its total population was situated southeast of the line, giving a population density of $135.39 / \mathrm{km}^{2}$ in 
that region, while $64 \%$ of the land area and $4 \%$ of the population was situated northwest of it, giving a population density of $5.03 / \mathrm{km}^{2}$. Due to changes in administrative borders, the proportion of China's land area in the northwest fell to $56.75 \%$ by 1953 , while that of the southeast increased to $43.24 \%$, but the proportion of population either side of the line remained largely similar, changing by approximately 1.5 percentage points. Between 1953 and 2010 , the proportion of China's population southeast of the line decreased slightly from $94.8 \%$ to $94.41 \%$, while that of the northwestern half increased slightly from $5.2 \%$ to $5.59 \%$. Population density, meanwhile, increased more significantly southeast and northwest of the line, from $139.51 / \mathrm{km}^{2}$ and $5.83 / \mathrm{km}^{2}$ in 1953 to $325.84 / \mathrm{km}^{2}$ and $14.68 / \mathrm{km}^{2}$ in 2010 , respectively.

\section{The unbreakable pattern of the Hu Huanyong Line and the solvable Premier's question}

\subsection{Unbreakable nature of the Hu Huanyong Line}

The last 80 years since the Aihui-Tengchong Line was first proposed has proven that, although China's total population increased from 458 million in 1935 to 1.36 billion in 2014, the spatial distribution has changed slightly and the proportion of China's population southeast of the line decreased from 96\% in 1933 to $94.41 \%$ in 2010, the Hu Huanyong Line has, nevertheless, been widely accepted and recognized as a baseline of China's population distribution, and population dense-sparse relationship and east-west pattern it outlines remain largely the same. It is thus a classic objective population distribution law of human and economic geography, and its unbreakable nature is reflected in the following three aspects.

\subsubsection{No change to the pattern of densely populated east and sparsely populated west}

Looking at the statistics from 1933 (Figure 2), it is evident that the distribution of China's population is very uneven, but this imbalance has gradually evolved through history, so no fundamental change is likely to take place to the pattern of having a dense population southeast of the line and a sparse population northwest of the line. The area southeast of the line accounts for approximately $43 \%$ of China's total land mass, but $95 \%$ of its total population, while the area northwest of the line accounts for approximately $57 \%$ of the total land mass, but $5 \%$ of the population. Population density in the northwest is well below that of the southeast. The increased proportion of the population living in the northwest can also be accounted for by the large number of ethnic minority groups living in the western region who were exempt from the one-child policy, which led to higher natural population growth in the west than in the east. This background mechanism is not the result of large-scale migration from east to west. Related studies on population distribution confirm this. Using county-level data from the fifth census, Ge Meiling and Feng Zhiming et al. produced an isopleth diagram of population density and a series of diagrams on China's population distribution, which showed that as China's population density has increased, population distribution has gradually shifted from southwest to east, with a tendency of movement from sparsely populated areas to densely populated ones. Their research confirmed that the distribution of China's population in 2000 was little changed from the distribution described by 
$\mathrm{Hu}$ in 1935, with more than three-quarters of the country's population still concentrated on one-fifth of China's land area and less than $2 \%$ of the population living in more than half of China's territory in 2000 (Ge and Feng, 2009).
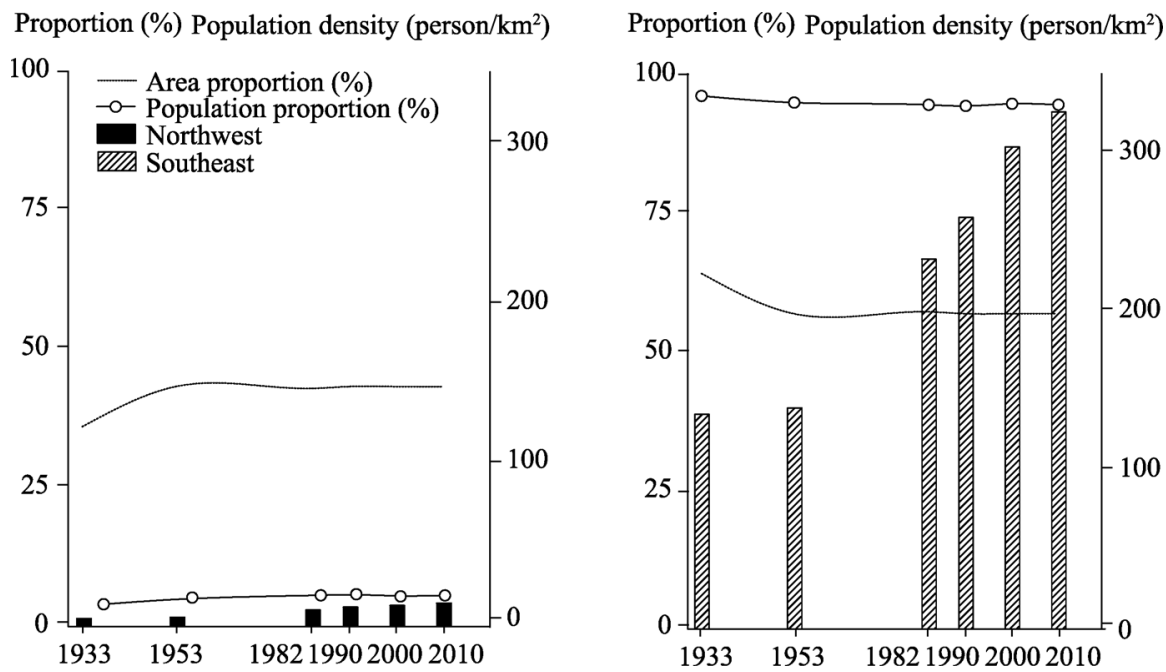

Figure 2 Changes of the proportion and density of population on northeast and southwest sides of the Hu Huanyong Line

4.1.2 No change also to the continued concentration of urban agglomerations southeast of the Hu Huanyong Line

Urban agglomerations are key areas of future economic growth and urbanization, and are necessarily places with favorable climate, topography, soil and water conditions. They are mostly situated along the coast in the east and in plains and basins in the west.

In the 19th century the sea was referred to as "the great road". Throughout history, the majority of human social and economic activities have taken place in coastal regions. In this globalized world, the most dynamic and economically competitive areas are urban agglomerations. The development advantages of coastal China have increased further, and the urban agglomerations in the Yangtze River Delta, Pearl River Delta and the Bohai Rim are becoming hubs through which China interacts with the world and the world interacts with China (Lu, 2003). The concentration of China's urban population in central and eastern regions will be an important urbanization trend for a period to come. This is not simply to do with the comparative advantages and geographic conditions of these regions, it is also an important way of balancing and concentrating the population and economy as well as narrowing the development gap (Fan et al., 2013a, 2013b). Figure 3 shows that the Yangtze River Delta, Pearl River Delta and Beijing-Tianjin-Hebei urban agglomerations are all located in coastal areas on the southeast side of the Hu Huanyong Line. These three urban agglomerations account for $28 \%$ of China's total population, and that proportion is continuing to grow. All of China's coastal provinces, meanwhile, account for $45 \%$ of the country's total population, a proportion that is also growing. With the aid of modern support systems, they can become high-efficient, energy-saving and modernized development spaces.

With the promotion of the Yangtze River Economic Belt to the status of a national strat- 
egy, based on overall resource and environmental carrying capacities, a number of cities along the river have grasped greater development opportunities. At the National Economic Geography and Land Planning Symposium held in Urumqi in 1984, Lu Dadao delivered a report entitled "Scientific Basis of a Diagram on China's Industrial Productivity Layout in 2000", in which he explained his T-shaped macroscopic strategy for regional development based on his theory of the "pole-axis" system, whereby the coastal area and Yangtze River Delta formed a national-level development axis (Lu, 2001; Liu and Lu, 2005). As China has entered a new normal of economic development, the construction of the Yangtze River Economic Belt has taken on extreme importance to the country, and a great deal of attention has been given to its development in the new period ( $\mathrm{Lu}, 2014)$, earning $\mathrm{Lu}$ the name of "father of the Yangtze River Economic Belt". The Yangtze River Economic Belt is a key region of strategic and guiding importance to national development. From east to west, it encompasses the Wanjiang urban agglomeration, Poyang Lake rim urban agglomeration, Wuhan Metropolitan Area, Changsha-Zhuzhou-Xiangtan urban agglomeration, and Chengdu-Chongqing urban agglomeration. The government has promised to provide capacity guarantees and scientific spatial organization in the areas of transport, IT, energy, urban development and domestic and foreign trade platforms.
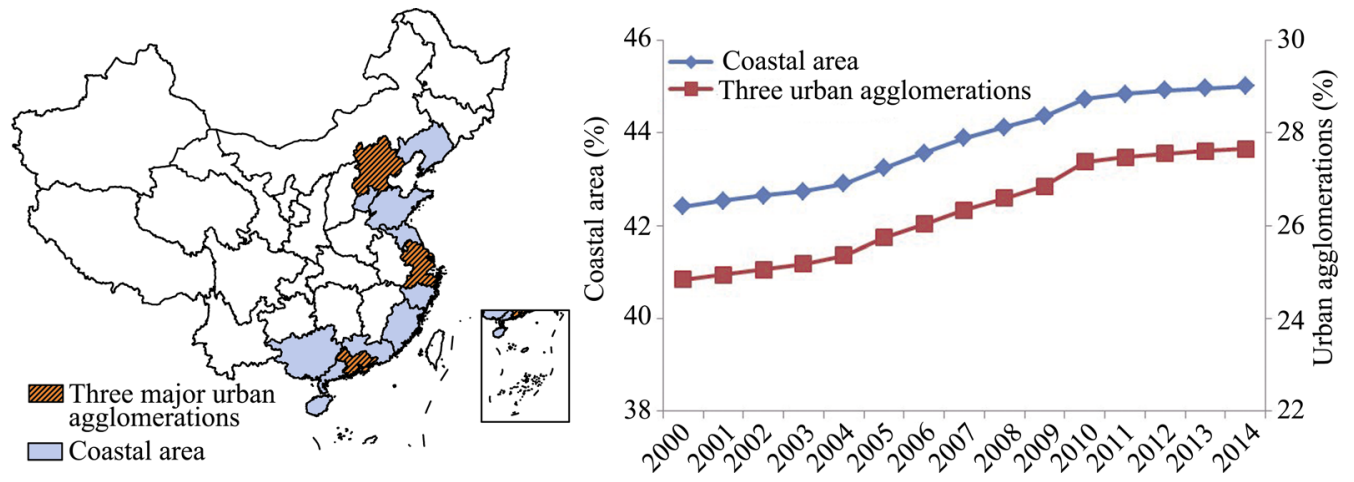

Figure 3 The change of urban agglomerations distribution and population proportion in coastal area of China Note: Coastal areas include Beijing, Tianjin, Hebei, Liaoning, Shanghai, Jiangsu, Zhejiang, Fujian, Shandong, Guangdong, Guangxi and Hainan. The three major urban agglomerations are the Beijing-Tianjin-Hebei, Yangtze River Delta and Pearl River Delta agglomerations, with calculations based on data from Beijing, Tianjin, Hebei, Shanghai, Zhejiang and Guangdong.

4.1.3 The integrated physical-geographic conditions as the determinant cause for the unbreakable of the Hu Huanyong Line

The stable pattern of population distribution either side of the Hu Huanyong Line has a significant geographical background and a series of climatic, topographic, cultural and economic determining factors. When interpreting differences in population distribution between east and west, $\mathrm{Hu}$ identified three factors: differences in the natural environment, levels of economic development, and social and historical conditions ( $\mathrm{Hu}, 1935)$. Obviously, the natural environment is the most fundamental factors of these three, while the latter two are influenced by the natural environment. As such, the Hu Huanyong Line is not just a demographic line; it is also an overall eco-environmental boundary (Wang and Zhang, 1995). 


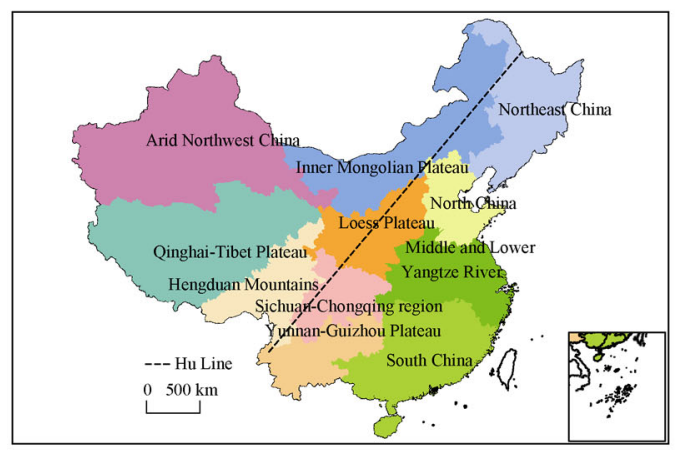

(a) Eco-regionalization

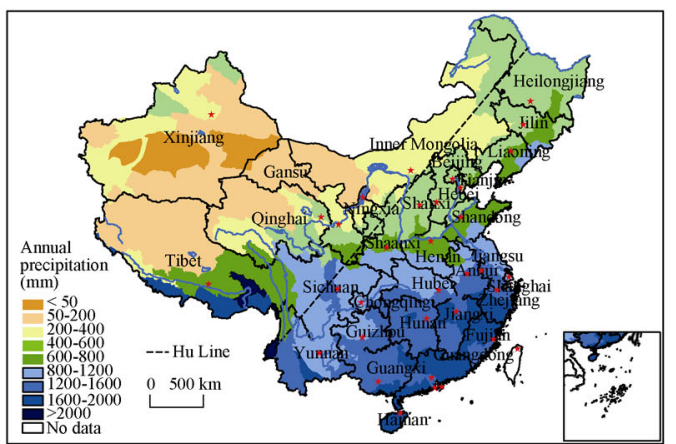

(c) Mean annual precipitation

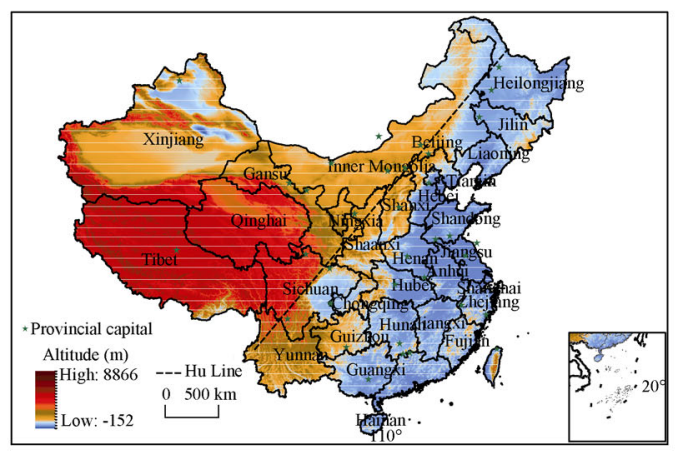

(b) Topographic classification

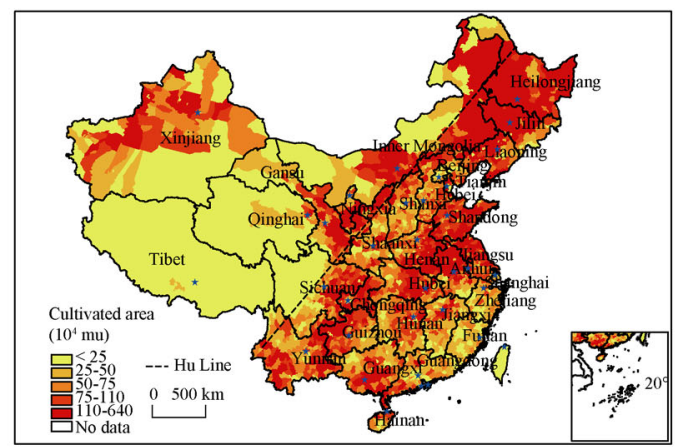

(d) Arable land distribution $(15 \mathrm{mu}=1 \mathrm{ha})$

Figure 4 Integrated physical factors for the formation of the $\mathrm{Hu}$ Huanyong Line

The Hu Huanyong Line runs along China's eco-environmental transition zone. China's ecological partitions are obvious (Xie et al., 2012): the area northwest of the line is characterized by harsh climatic conditions and consists of grasslands, deserts and snow-covered plateaus, which have been home to nomads since ancient times; the area southeast of the line, on the other hand, is hot and humid and consists of plains, networks of rivers and lakes, karst topography and Danxia landforms, with an economic foundation in agriculture also stretching back to ancient times (Figure 4a). Therefore, the Hu Huanyong Line is not just a dividing line of population distribution, it is also seen as the dividing line between China's different eco-environments. It is a gradient zone for the transition between changing ecological conditions. China's fragile eco-environmental areas, including areas prone to landslides, debris flows, earthquakes and other geomorphologic hazards, are located along the $\mathrm{Hu}$ Huanyong Line. It also divides the non-flooding west from the flood-prone east.

The Hu Huanyong Line runs along China's topographical transition zone. Approximately $80 \%$ of the world's population lives below an altitude of $500 \mathrm{~m}$, and China's population has a noticeable vertical distribution. More than $70 \%$ of the population lives below an altitude of $500 \mathrm{~m}$, with $50 \%$ living below an altitude of $200 \mathrm{~m}$. It can clearly be seen from Figure $4 \mathrm{~b}$ that China is low-lying southeast of the Hu Huanyong Line and more elevated northwest of it. Thus, the division of China's topography roughly corresponds to the Hu Huanyong Line for population density. From a quantitative study, Feng Zhiming et al. discovered that a strong correlation exists between China's topographic undulating degree and its population density, with a logarithmic score of 0.91 between the two (Feng et al., 2007).

The Hu Huanyong Line runs along China's climatic transition zone. As can be seen from 
Figure 4c, the line broadly coincides with the $400 \mathrm{~mm}$ isohyet, which divides China's semi-arid and semi-humid regions. Areas southeast of the line enjoy abundant rainfall, whereas those northwest of the line receive less than $400 \mathrm{~mm}$. Research has shown that central and northern parts near the Hu Huanyong Line are on the terminus affected by the southeast monsoon, and southwestern parts near the line are the western limit affected by the southwest monsoon. As such, it could be said that the formation of the Hu Huanyong Line was also caused by rainfall differences related to climate.

As can be seen from Figure 4, east of the Hu Huanyong Line, including China's northeast, north, center and southeast, mostly consists of plains and hilly terrain, whereas west of the line, including China's west and northwest, consists of the Qinghai-Tibet Plateau, Hengduan Mountains, Inner Mongolia Plateau and northwest desert. Specific natural conditions greatly restrict the industries local populations are engaged in, with people east of the line mainly engaged in agriculture, while those west of it are mainly engaged in animal husbandry. The central part consists of key sediment producing areas, including the Loess Plateau, a major source of the sedimentation of the Yellow River. The Qinghai-Tibet Plateau, semi-arid areas, farming-pastoral zones, areas of karst topography and other special areas are largely ecologically fragile areas, some with severe soil and water shortages. It is generally almost impossible to carry out large-scale industrialization and urbanization in these areas. Thus, there is, to some extent, an overlap between the Hu Huanyong Line and the boundaries of China's topography, precipitation, ecological areas, cultural landscapes and nationalities, and the demographic pattern of a dense population southeast of the Hu Huanyong Line and a sparse population northwest of it is also the product of the long-term combined effects of nature, resources, humans and the economy. People choose places suitable for farming, developing and reproducing. From the diagram of distribution of China's arable land (Figure 4d), it can be seen that it is mostly located southeast of the Hu Huanyong Line. According to a survey of changing land use by the Ministry of Land and Resources, there is 431.57 million $m u$ (28.78 million ha) of arable land northwest of the $\mathrm{Hu}$ Huanyong Line, accounting for $23.64 \%$ of the country's total, and 1.394 billion $m u$ (92.97 million ha), accounting for $76.36 \%$ of the total, southeast of the line.

\subsection{The "solvable" part of the Premier's question: A new-type urbanization in China's western region}

The above analysis has shown that there is no solution to the Premier's question of how China can break with the traditional demographic pattern of the Hu Huanyong Line. But if we look at the Premier's prime concern when he raised the issue, we find that there is a solution. That is because the Premier was actually concerned with getting people in central and western regions to benefit from urbanization and modernity. In this respect, the central region is doing quite well, but the western region presents greater difficulties. As space is limited, our focus is on analyzing a new-type urbanization for the western region. We believe that, on the basis of adhering to the objective law of the Hu Huanyong Line, it is entirely possible to achieve higher levels and higher quality urbanization in the central and western regions through rational population, urban and industrial planning.

\subsubsection{Accelerating properly urbanization of the western region}

Since entering a period of urbanization, the western region has also entered a stage of rapid 
development. Between 2006 and 2012, the level of urbanization in the western region has increased from $35.69 \%$ to $44.74 \%$, an average increase of 1.5 percentage points, giving it an urbanization rate above the national average and higher than the previous period. At the same time, studies have revealed a Matthew effect between the urbanization and economic development levels of various provinces, with the urbanization levels of the economically underdeveloped central and western regions lagging behind their economic development. Therefore, the process of urbanization could be accelerated appropriately in some provinces of the central and western regions. The construction of national-level new areas, such as the Liangjiang New Area in Chongqing, Lanzhou New Area in Gansu, Xixian New Area in Shaanxi, Gui'an New Area in Guizhou and Tianfu New Area in Chengdu, provide experimental zones for the scientific development of the western region. The construction of major infrastructure projects is promoting links between regions and cities and strengthening the supporting and guiding role of transport toward urbanization. In the 13th Five-Year Plan period, the western region remains in a period of rapid urbanization, and it should seize opportunities to actively pioneer a new regional model of urbanization with its own characteristics.

The western region is faced with multiple overlapping advantages in urbanization. The first is the release of the "National New-type Urbanization Plan". The plan was announced in March 2014, and it proposed comprehensively improving the quality of urbanization by accelerating transformation of the mode of urbanization and pursuing a new-type of urbanization with Chinese characteristics that puts people first, places equal emphasis on industrialization, agricultural modernization, urbanization, and the application of IT, optimizes the overall layout of cities, is environmentally sound, and preserves China's cultural heritage. It also proposed guiding the movement of about 100 million people to their nearest cities in central and western regions. The second advantage is national strategies that include the Silk Road Economic Belt and the Yangtze River Economic Belt. In September 2013, Chinese President Xi Jinping delivered an important speech at Nazarbayev University in Kazakhstan, in which he proposed establishing the Silk Road Economic Belt. In June of the following year, Premier Li Keqiang chaired an executive meeting of the State Council where he announced the plan to establish a comprehensive three-dimensional transportation corridor to create the Yangtze River Economic Belt. The two national strategies together will help bring western border regions from the periphery to the forefront of China's opening up and create new internationally influential platforms of opening up and cooperation.

4.2.2 Helping rural migrant workers to become urban residents and promoting better quality urbanization

The "National New-type Urbanization Plan" clearly proposed to improve the quality of urbanization and make helping migrant workers to become urban residents a key task. The plan also pointed out the need to create the conditions for urbanization and then letting it happen organically rather than forcing people to move, and for local governments to play down urbanization growth indicators and not classify them as binding assessment indicators.

At the core of the new-type urbanization path is the solid and orderly promotion of migrant workers to become city dwellers. In accordance with the principles of respecting people's wishes, individual choices, local conditions, incremental progress, prioritizing efforts to help existing migrant workers become permanent city dwellers, by which new migrant 
workers will also gradually be integrated into cities, and with the focus on promoting rural migrant workers to become urban residents, coordinated efforts will be made to promote reform of the household registration system and make public service provisions more equal. With this in mind, reform and classification of the household registration system will be accelerated, with overall consideration given to the size of regional (prefectual, district) urban populations and resource and environmental carrying capacities and helping migrant workers to become urban residents in various tiers of towns and cities. The focus will be on the differences between the registered population urbanization rate and the resident population urbanization rate and gradually narrowing the statistical difference between the two. An assessment will also be made of access to basic public services for rural migrant workers who have moved to cities. The ability of towns and cities to absorb the floating population will be enhanced in order to inject vitality into local economic and social development.

Guiding migrant workers to nearby towns and cities involves accepting migrant workers returning from eastern and central regions, local agricultural workers and casual migrant workers from elsewhere, with consideration given to college vocational and technical school graduates, the unemployed from other towns and cities, and city and suburban agricultural populations. Promoting migrant workers gaining access to urban basic public services mainly includes dependents of people who move to cities having the equal right to education, being covered by social security and having access to basic medical and health care and housing.

4.2.3 Developing labor-intensive industries and the service industry to achieve a higher level of modernization

Many provinces have introduced policies to promote eligible rural migrant workers becoming urban residents, with average results. The main reason for this is that the factor restricting western region migrant workers becoming urban residents is not the administrative system, but rather the inability of local industries to provide adequate employment. Therefore, in future it will be necessary to strongly support the development of labor-intensive enterprises, the service industry and micro-enterprises, as well as to promote the shift of labor-intensive industries from China's eastern to the western region.

Looking at the current transfer of industries, market capital is moving toward resource-based industries in the western region, while the transfer of labor-intensive industries is happening relatively slowly. The government should provide appropriate policy support for labor-intensive industries to move west and promote coordinated industrial development and urbanization. Life service industries capable of generating large numbers of jobs and with significant labor absorptive capacity urge industrial cities to gradually become service cities. Small and medium-sized cities and small towns should focus on developing competitive industries and labor-intensive industries and vigorously support agro-industries and producer services to rural farmers.

The western region should adopt an urbanization path that involves coordinating the development of large, medium-sized and small cities and small towns. Different types and tiers of cities each have their own status, functions and roles, and development between cities can be mutually restraining or mutually promoting. Small and medium-sized cities in the western region suffer from a lack of vitality. There are obvious advantages to small and medium-sized cities and small towns promoting migrant workers to become urban residents, 
including low barriers to entry, particularly in terms of the cost of living and the low cost of housing and social costs. Moreover, small and medium-sized cities and small towns develop by attracting rural migrant workers, and increasing the size of cities gives play to the effectiveness of infrastructure investment and allows the better provision of public services.

\subsubsection{A development path of concentrated population flows for the western region}

With the accelerated pace of urbanization in the western region, and as migrant workers return from central and eastern regions, it is recommended that they are guided to concentrate in urban agglomerations with relatively large environmental carrying capacities, but disperse in small and medium-sized cities away from central cities in order to avoid excessively overloading central megacities and to direct greater concentrations toward cities on the edges of agglomerations.

Greater concentration is recommended so as to improve the overall carrying capacity of urban agglomerations in the western region. Urban agglomerations in the west are still taking shape and growing, and in addition to national policies for actively guiding their formation, other factors promoting their rapid development include relatively good resource and environmental bases, location advantages, transport advantages, industrial bases and relatively concentrated populations. During the 13th Five-Year Plan, efforts should be made to further improve the overall carrying capacities of urban agglomerations in the western region, to guide people and industries to concentrate in urban agglomerations, to improve the overall carrying capacities of cities, to develop and expand urban agglomerations, and to increase the proportions of their populations and economies. Of course, promotion of their development should accord with local characteristics and should not be based on blind comparisons with urban agglomerations in the east. The western region is not suitable for large-scale megalopolises, so it must implement an urbanization model of concentrated, tight-knit urban areas and promote intensive, concentrated and centralized urbanization.

Dispersal within urban agglomerations mainly involves guiding migrant workers returning from eastern and central regions as well as local agricultural workers moving to cities to settle in small and medium-sized cities in urban agglomerations. It also involves expanding border towns and cities to stabilize the populations of border areas and help them develop and prosper.

4.2.5 Building the "Three Horizontals and One Vertical" multi-level urban spatial structure and optimizing spatial organization

Using a spatial optimization strategy centered on a pole-axis system, a group structure and bead-style expansion, a multi-level urban system spatial structure consisting of three horizontal and one vertical line of urban agglomerations should be created (Figure 5). It is necessary to strengthen the first-tier axis of urbanization formed by the Baotou-Kunming railway, Lianyungang-Alataw Pass railway, "Golden Waterway" of the Yangtze River, Xijiang railway and other transportation axes; enhance the role of international gateway cities; give play to the leading development demonstration effect of new areas; foster the growth of the Chengdu-Chongqing urban agglomeration and the Guanzhong-Tianshui urban agglomeration; form a pattern based on urban agglomerations; organize division of labor within urban agglomerations; strive to concentrate population, economies and industries; further improve the development and vitality of small and medium-sized cities; select key development 
towns; respect natural and geographical restraints in border areas, cold areas and oasis towns; and adopt a beaded or dotted distribution of towns.

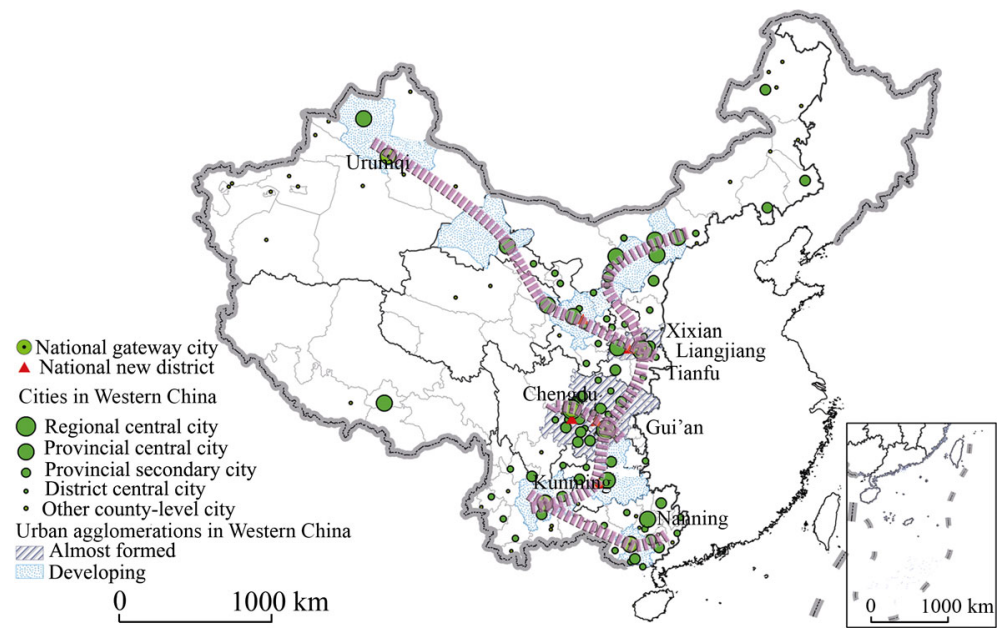

Figure 5 "Three Horizontals and One Vertical" spatial layout of the multi-level urban system in western China

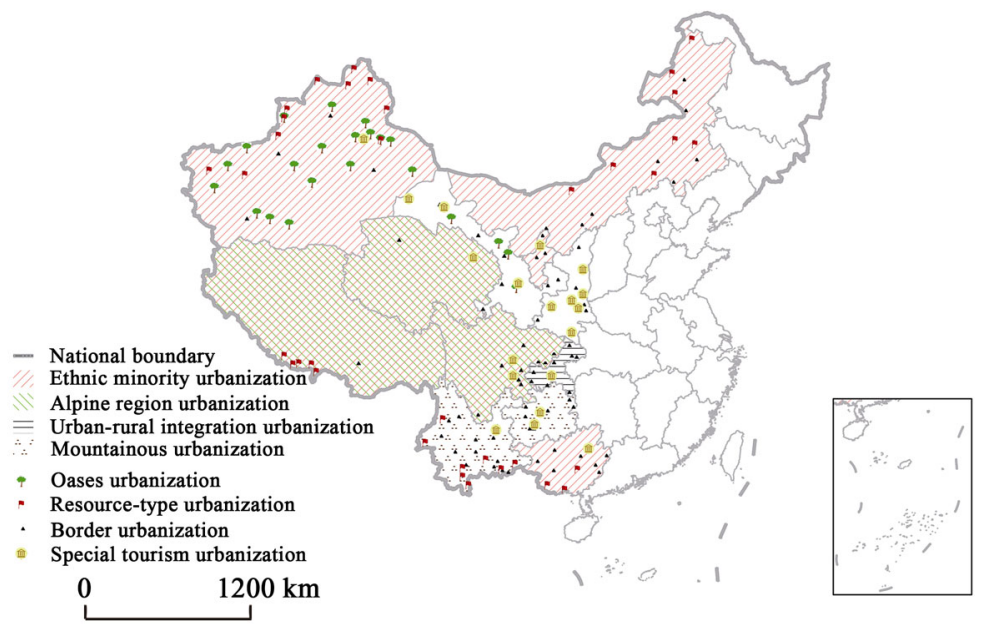

Figure 6 Characteristic modes of Chinese western urbanization

The three horizontal axes refer to the transport corridors of the Lianyungang-Alataw Pass railway, Yangtze River "Golden Waterway" and Xijiang railway, which are east-west axes of urbanization. The one vertical axis refers to the Kunming-Baotou railway, which starts in Baotou and Hohhot and passes through Xi'an, Chongqing, and terminates in Kunming and Nanning, and is an important gathering area of population and urbanization in the western region. It is multi-level in that it encompasses various tiers of urbanized entities, including international gateway cities, important new areas, urban agglomerations, provincial capitals, small and medium-sized cities and small towns. It is necessary to integrate local conditions, select an urbanization model and path suited to local conditions and explore numerous new-type regional urbanization and development models, for example models for the urbanization of oases, mountainous areas, ethnic minority areas and border areas (Figure 6). 
Different development models will have certain overlapping features in terms of space.

\section{Conclusions and discussion}

(1) The pattern of population distribution illustrated by the Hu Huanyong Line is an objective law of human and economic geography, which has a profound geographical background. This pattern cannot be "broken", nor is there any need to do so. Hu Huanyong stated in his 1935 study, "People unfamiliar with the geographical facts know China's southeast to be densely populated and the western provinces to be sparsely populated, and therefore assume that there is great potential for transplanting development. They are unaware that this is not actually the case. Today, the northwest... is made up of plains and basins with small and narrow habitable areas, and it has a dry climate, with water only available in the form of snowmelt from the high mountains, from man-made canals and from exceptionally deep wells...." (Hu, 1935). This is still applicable today. Of course, there is the possibility of the demographic pattern of the Hu Huanyong Line being broken in the future, for example by a dramatic change in climatic conditions due to global warming.

(2) Premier Li Keqiang's question is "solvable". The solution given here is not to attempt to break the demographic pattern of the Hu Huanyong Line in a real sense, but to respond to the heart of the Premier's question, which was how to promote the new-type urbanization in the central and western regions to allow people there to enjoy the benefits of urbanization and modernization. We believe that active policy guidance and rational spatial organization can allow the central and western regions to achieve fully a higher level of modernization and better quality urbanization. This study has attempted to construct a new urbanization idea and path for the western region.

(3) The demographic pattern of a densely populated east and sparsely populated west and the hope of achieving a higher level of urbanization in the western region are not contradictory. They can be coordinated and simultaneously achieved. Overall consideration needs to be given to the resource and environmental carrying capacities and the economic and social development potential of different places, as the most likely future scenario is a greater concentration of population in central and eastern regions. Those that can may settle in central and eastern towns and cities, while some will settle in towns and cities in the western region that have relatively good resource and environmental carrying capacities. These two methods arrive at the same end by different means, as they both help increase the level and quality of urbanization in the western region.

(4) There is currently considerable social awareness about the Hu Huanyong Line and "the Premier's question", but there is still an urgent need for more scientific research to support relevant decision making by the government. This study has simply constructed a conceptual model to analyze the ongoing relevance of the Hu Huanyong Line at the macroscopic level. In future there is a need to strengthen quantitative analyses of the coupling relationship between physical geographic factors and population and economy so as to reveal the formation conditions and historical evolution of the Hu Huanyong Line.

\section{References}

Ding Jinhong, Liu Zhenyu, Cheng Danming et al., 2005. Areal differentiation of inter-provincial migration in China and characteristics of the flow field. Acta Geographica Sinica, 60(1): 106-114. (in Chinese)

Fan Jie, Liu Yi, Chen Tian et al., 2013a. The key strategies and innovative thinking for optimization on spatial 
pattern of urbanization in China. Bulletin of Chinese Academy of Sciences, 28(1): 20-27. (in Chinese)

Fan Jie, Wang Qiang, Zhou Kan et al., 2013b. Preliminary research on spatial pattern of urbanization in mountainous areas of China. City Planning Review, 37(5): 9-15. (in Chinese)

Feng Zhiming, Tang Yan, Yang Yanzhao et al., 2007. The relief degree of land surface in China and its correlation with population distribution. Acta Geographica Sinica, 62(10): 1073-1082. (in Chinese)

Feng Zhiming, Yang Yanzhao, You Zhen et al., 2014. Research on the suitability of population distribution at the county level in China. Acta Geographica Sinica, 69(6): 723-737. (in Chinese)

Ge Meiling, Feng Zhiming, 2008. Research on distribution pattern of population of China in 2000 based on GIS: Compared with Hu Huanyong's research in 1935. Population Research, 32(1): 51-57. (in Chinese)

Ge Meiling, Feng Zhiming, 2009. Population distribution of China based on GIS: Classification of population densities and curve of population gravity centers. Acta Geographica Sinica, 64(2): 203-210. (in Chinese)

Hu Huanyong, 1935. The distribution of population in China, with statistics and maps. Acta Geographica Sinica, 2(2): 33-74. (in Chinese)

Jia Kang, Su Jingchun, 2015. Hu Line: "Half pressure type" of environmental pressure and targeted management strategy in China. Journal of the Party School of the Central Committee of the C.P.C., 19(1): 64-75. (in Chinese)

Jia Shaofeng, 2014. Premier Li, it is unworkable to break Hu Line! http://blog.sciencenet.cn/blog-267937-850831. html, 2014-12-14. (in Chinese)

Kuang Xianming, 2014. One Belt One Road: A way to break Hu Line. Shenzhen Special Zone Daily. http://sztqb.sznews.com/html/2014-12/01/content 3079606.htm, 2014-12-01. (in Chinese)

Li Peilin, 2015. New urbanization and the break of Hu Line. People's Daily. http://theory.people.com.cn/n/ 2015/0108/c40531-26347255.html, 2015-01-18. (in Chinese)

Liu Guixia, 2004. The origin of Aihui-Tengchong population line. Map, (6): 48-51. (in Chinese)

Liu Jingsong, 2014. A review of population geography research in China. Acta Geographica Sinica, 69(8): 1177-1189. (in Chinese)

Liu Weidong, Lu Dadao, 2005. Methodological basis for making regional spatial planning in China in the new era. Acta Geographica Sinica, 60(6): 894-902. (in Chinese)

Liu Yanhua, Wang Qiang, 2001. Optimized population distribution in China: On provincial sustainable development. China Population, Resources and Environment, 11(1): 34-37. (in Chinese)

Lu Dadao, 2001. An analysis of spatial structure and optimal regional development. Acta Geographica Sinica, 56(2): 127-135. (in Chinese)

Lu Dadao 2003. New factors and new patterns of regional development in China. Geographical Research, 22(3): 261-271. (in Chinese)

Lu Dadao, 2011. Development of geographical sciences and research on global change in China. Acta Geographica Sinica, 66(2): 147-156. (in Chinese)

Lu Dadao, 2014. Economic belt construction is the best choice of economic development layout. Scientia Geographica Sinica, 34(7): 769-772. (in Chinese)

Shen Jianfa, 2005. Analysis of the trends of urbanization levels in Chinese provinces since 1982. Acta Geographica Sinica, 60(4): 607-614. (in Chinese)

Tien, H. Y., 1973. China's Population Struggle: Demographic Decisions of the People's Republic of China, 1949-1969. Ohio State University Press.

Tien, H. Y., 1981. Demography in China: From zero to now. Population Index, 47(4): 683-710.

Wang Guixin, 1997. China's population distribution and regional economic development. Shanghai: East China Normal University Press, 200-201. (in Chinese)

Wang Jingai, 1998. The pattern of China's population distribution and the sustainable development. Journal of Beijing Normal University (Social Sciences), (1): 72-80. (in Chinese)

Wang Zheng, 2014. Two keys to break Hu Line. Wenhui Daily, http://www.qstheory.cn/zhuanqu/ bkjx/ 2014-12/02/c_1113487608.htm, 2014-12-02. (in Chinese)

Wang Zheng, Zhang Peiyuan, 1995. On the ecological sensitive zone in China. Acta Ecologica Sinica, 15(3): 319-326. (in Chinese)

Wu Chuanjun, 2001. Contributions of master Hu Huanyong to the development of modern geography in China. Human Geography, 16(5): 1-4. (in Chinese)

Wu Jing, Wang Zheng, 2008. Agent-based simulation on the evolution of population geography of China during the past 2000 years. Acta Geographica Sinica, 63(2): 185-194. (in Chinese)

Xie Gaodi, Zhang Changshun, Zhang Linbo et al., 2012. China's county-scale ecological regionalization. Journal of Natural Resources, 27(1): 154-162. (in Chinese)

Yang Fang, 2014. Premier Li Keqiang's question: How to break Hu Line? http://www.gov.cn/xinwen/2014-11/29/ content_2784676.htm, 2014-11-29. (in Chinese)

Zhu Yu, 2006. New forms of urbanization and China's urbanization policies. Human Geography, 21(2): 115-118, 128. (in Chinese) 